\title{
MODULES SATISFYING THE PRIME AND MAXIMAL RADICAL CONDITIONS
}

\author{
MAHMOOD BEHBOODI AND MASOUD SABZEVARI
}

\begin{abstract}
In this paper, we introduce and study $\mathbb{P}$ radical and $\mathbb{M}$-radical modules over commutative rings. We say that an $R$-module $M$ is $\mathbb{P}$-radical whenever $M$ satisfies the equality $(\sqrt[p]{\mathcal{P} M}: M)=\sqrt{\mathcal{P}}$ for every prime ideal $\mathcal{P} \supseteq \operatorname{Ann}(\mathcal{P} M)$, where $\sqrt[p]{\mathcal{P} M}$ is the intersection of all prime submodules of $M$ containing $\mathcal{P} M$. Among other results, we show that the class of $\mathbb{P}$-radical modules is wider than the class of primeful modules (introduced by $\mathrm{Lu} \mathrm{[19]).} \mathrm{Also,} \mathrm{we}$ prove that any projective module over a Noetherian ring is $\mathbb{P}$-radical. This also holds for any arbitrary module over an Artinian ring. Furthermore, we call an $R$-module $M$ by $\mathbb{M}$ radical if $(\sqrt[P]{\mathcal{M M}}: M)=\mathcal{M}$, for every maximal ideal $\mathcal{M}$ containing Ann $(M)$. We show that the conditions $\mathbb{P}$-radical and M-radical are equivalent for all $R$-modules if and only if $R$ is a Hilbert ring. Also, two conditions primeful and $\mathbb{M}$-radical are equivalent for all $R$-modules if and only if $\operatorname{dim}(R)=0$. Finally, we remark that the results of this paper will be applied in a subsequent work of the authors to construct a structure sheaf on the spectrum of $\mathbb{P}$-radical modules in the point of algebraic geometry view.
\end{abstract}

1. Some preliminaries. For an arbitrary commutative ring $R$, the associated spectrum Spec $(R)$ of $R$ is the family of its prime ideals. Let $V(I)=\{\mathcal{P} \in \operatorname{Spec}(R): I \subseteq \mathcal{P}\}$ for each ideal $I$ of $R$. One can equip Spec $(R)$ with the so-called Zariski topology by considering the sets of the form $V(I)$ as the closed subsets of this topology. Moreover, it is proved that the collection $\{D(f), f \in R\}$ is a basis for this

2010 AMS Mathematics subject classification. Primary 13C13, 13C99, 13A99, $14 \mathrm{~A} 25$.

Keywords and phrases. Prime submodule, prime spectrum, $\mathbb{P}$-radical module, Zariski topology, sheaf of rings, sheaf of modules.

The research of the authors was in part supported by grants from IPM (Nos. 92130413 and 91530040). This research was partially carried out in the IPM-Isfahan Branch. The second author also was partially supported by the Center of Excellence for Mathematics, University Of Shahrekord, Iran.

Received by the editors on September 25, 2012. 
topology where $D(f)=\{\mathcal{P} \in \operatorname{Spec}(R): f \notin \mathcal{P}\}=\operatorname{Spec}(R) \backslash V(f)$ and $V(f)=V(R f)($ see $[\mathbf{2}, \mathbf{1 5}])$.

All rings in this article are commutative with identity and modules unital. For a $\operatorname{ring} R$, we denote by $\operatorname{dim}(R)$ the classical Krull dimension of $R$, and for a submodule $N$ of an $R$-module $M$ we denote the annihilator of the factor module $M / N$ by $(N: M)$, i.e., $(N: M)=$ $\{r \in R \mid r M \subseteq N\}$. $M$ is called faithful if $(0: M)=0$. A non-zero $R$-module $M$ is called prime if the equality $r m=0$ for $m \in M ; r \in R$ implies that $m=0$ or $r M=(0)$ (i.e., $r \in \operatorname{Ann}(M)$ ). We recall that a proper submodule $P$ of $M$ is a prime submodule if $M / P$ is a prime module (i.e., for every $r \in R$ and $m \in M$, if $r m \in P$, then $m \in P$ or $r \in(P: M))$. In this case, $\mathcal{P}=(P: M)$ is a prime ideal of $R$ and $M / P$ is a torsion free $R / \mathcal{P}$-module. This motivates one to call the prime submodule $P$ by $\mathcal{P}$-prime submodule.

This notion of prime submodule was firstly introduced and systematically studied by Dauns [9] and Feller and Swokowski [12] and recently has received a good deal of attention from several authors (see for instance, $[4,6,7,16,20,21,22,24])$. Motivated by algebraic geometry, the set of all prime submodules of $M$ is called the spectrum of $M$ and is denoted by $\operatorname{Spec}(M)$. Similar to the case of commutative rings, the sets of the form:

$$
V(N)=\{P \in \operatorname{Spec}(M) \mid(N: M) \subseteq(P: M)\},
$$

for any arbitrary submodule $N$ of $M$ allow one to associate the Zariski topology on the collection $\operatorname{Spec}(M)$, in which each of the sets $V(N)$ is a closed subset in this topology (see for instance, $[\mathbf{1 7}, \mathbf{1 8}, \mathbf{2 3}, \mathbf{2 6}]$ ).

For an $R$-module $M$, consider the so-called natural map:

$$
\begin{aligned}
\psi: \operatorname{Spec}(M) & \longrightarrow \operatorname{Spec}(R / \operatorname{Ann}(M)) \\
P & \longmapsto(P: M) / \operatorname{Ann}(M) .
\end{aligned}
$$

An $R$-module $M$ is called primeful if either $M=(0)$ or $M \neq(0)$ and the associated natural map $\psi$ is surjective. This notion of primeful module has been introduced for the first time and extensively studied by Lu in [19]. She found out some worthwhile properties of this type of module. This motivated us to extend the class of primeful modules to wider classes (which we call $\mathbb{P}$-radical modules and $\mathbb{M}$-radical modules) in which the above-mentioned worthwhile properties are to be preserved. 
In fact, in the subsequent results (see [1]), we will show that this class of $\mathbb{P}$-radical module is an appropriate one for considering in an algebraic geometry view.

In Section 2, we introduce and study $\mathbb{P}$-radical modules and compare them with primeful modules. In particular, we show that the class of $\mathbb{P}$-radical modules is wider than that of primefuls (Proposition 2.3). Even more, we prove that any projective module over a Noetherian ring is also a $\mathbb{P}$-radical (Theorem 2.5). This also holds for any arbitrary module over an Artinian ring (Theorem 2.13). Furthermore, we call an $R$-module $M$ by $\mathbb{M}$-radical if $(\sqrt[p]{\mathcal{M} M}: M)=\mathcal{M}$, for every maximal ideal $\mathcal{M}$ containing $\operatorname{Ann}(M)$, where $\sqrt[p]{\mathcal{M} M}$ is the intersection of all prime submodules of $M$ containing $\mathcal{M} M$. We see the following chart of implications for $M$ :

$M$ is finitely generated $\Rightarrow M$ is primeful $\Rightarrow M$ is $\mathbb{P}$-radical $\Rightarrow M$ is $\mathbb{M}$-radical

We show that the two conditions $\mathbb{P}$-radical and $\mathbb{M}$-radical are equivalent for all $R$-modules if and only if $R$ is a Hilbert ring (Theorem 2.11). Also we see that the two conditions primeful and $\mathbb{M}$-radical are equivalent for all $R$-modules if and only if $\operatorname{dim}(R)=0$ (Theorem 2.12). Recall that an $R$-module $M$ is called a multiplication module if, for every submodule $N$ of $M$, there exists an ideal $I$ of $R$ such that $N=I M$ $($ see $[\mathbf{3}, \mathbf{1 1}]$ for more details). We prove that for a multiplication module $M$ the four conditions of the above table are equivalent (Proposition 2.18). Moreover, we give an analogue of Nakayama's lemma for $\mathbb{P}$-radical modules at the end of Section 2. In Section 3, semisimple primeful modules, semisimple $\mathbb{P}$-radical modules and semisimple $\mathbb{M}$ radical modules are fully investigated. For instance, in Proposition 3.5, it is shown that a semisimple $R$-module $M$ is $\mathbb{M}$-radical if and only if there exists a submodule $N$ of $M$ such that:

$$
N \cong \bigoplus_{\operatorname{Ann}(M) \subseteq \mathcal{M} \in \operatorname{Max}(R)} R / \mathcal{M} .
$$

Also, a semisimple $R$-module $M$ is $\mathbb{P}$-radical if and only if $M$ is a $\mathbb{M}$-radical and $R / \operatorname{Ann}(M)$ is a Hilbert ring (Proposition 3.6).

In [1], we will employ the results achieved in this paper to study the spectrum of modules from the point of view of algebraic geometry. In particular, we will construct a structure sheaf on the spectrum of the modules which belong to the wide class of $\mathbb{P}$-radicals (see also [26]). 
2. P-radical and $\mathbb{M}$-radical modules. Contrary to the rings with identity, one should notice that not every $R$-module contains a prime submodule. For example, $\mathbb{Z}_{p \infty}$ as a $\mathbb{Z}$-module does not contain any prime submodule (see $[4, \mathbf{1 2}])$. For a proper submodule $N$ of an $R$-module $M$, the prime radical $\sqrt[p]{N}$ is the intersection of all prime submodules of $M$ containing $N$. We put $\sqrt[p]{N}=M$ in the case that there is no such prime submodule. Clearly $V(N)=V(\sqrt[p]{N})$. We note that, for each ideal $I$ of $R, \sqrt[p]{I}=\sqrt{I}$ (the intersection of all prime ideals of $R$ containing $I$ ). The prime radicals of submodules are studied by several authors (see for instance, $[\mathbf{5}, \mathbf{6}, \mathbf{2 0}]$ ).

In [20], it is probed whether or not the equality $\sqrt[p]{I M}=\sqrt{I} M$ is satisfied for every ideal $I$ containing Ann $(M)$ in the case of finitely generated $R$-modules $M$. Also, in [19], the author extended the investigation to primeful flat content modules (e.g., free modules), and primeful flat modules over rings with Noetherian spectrum.

In this article, we introduce a slight differentiation of the above equality, that is:

$$
(\sqrt[p]{I M}: M)=\sqrt{I}
$$

for every ideal $I$ containing $\operatorname{Ann}(M)$. This radical condition on modules plays a key role in our work to build a desired structure sheaf of modules in [1]. The following proposition offers several characterizations of $R$-modules $M$ which satisfy the above condition.

Proposition 2.1. For an $R$-module $M$, the following four statements are equivalent:

(1) $(\sqrt[p]{I M}: M)=\sqrt{I}$ for every ideal $I \supseteq$ Ann $(M)$.

(2) $(\sqrt[p]{\mathcal{P} M}: M)=\mathcal{P}$ for every prime ideal $\mathcal{P} \supseteq \operatorname{Ann}(M)$.

(3) $\sqrt{I}=\bigcap_{P \in V(I M)}(P: M)$, for every ideal $I \supseteq \operatorname{Ann}(M)$.

(4) $\mathcal{P}=\bigcap_{P \in V(\mathcal{P} M)}(P: M)$, for every prime ideal $\mathcal{P} \supseteq$ Ann $(M)$.

Proof. (1) $\Rightarrow(2)$ and $(3) \Rightarrow(4)$ are trivial.

For $(2) \Rightarrow(1)$, let $I \supseteq$ Ann $(M)$. We have $I \subseteq(I M: M) \subseteq(\sqrt[p]{I M}$ : $M)$ and $(\sqrt[p]{I M}: M)$ an intersection of all prime ideals. This implies 
that $\sqrt{I} \subseteq(\sqrt[p]{I M}: M)$. On the other hand, we have:

$$
(\sqrt[p]{I M}: M) \subseteq \bigcap_{\mathcal{P} \in V(I)}(\sqrt[p]{\mathcal{P} M}: M)=\bigcap_{\mathcal{P} \in V(I)} \mathcal{P}=\sqrt{I},
$$

which immediately gives the desired equality $(\sqrt[p]{I M}: M)=\sqrt{I}$.

For $(1) \Rightarrow(3)$, one checks that, for each $I \supseteq \operatorname{Ann}(M)$, we have:

$$
\begin{aligned}
\sqrt{I} & =(\sqrt[p]{I M}: M)=\left(\left(\bigcap_{P \in V(I M)} P\right): M\right) \\
& =\bigcap_{P \in V(I M)}(P: M),
\end{aligned}
$$

as desired.

For $(4) \Rightarrow(2)$, suppose that $\mathcal{P} \supseteq \operatorname{Ann}(M)$ is a prime ideal of $R$. Substituting $\bigcap_{P \in V(\mathcal{P} M)} P$ with $\sqrt[p]{\mathcal{P} M}$ in the equality $\bigcap_{P \in V(\mathcal{P} M)}(P$ : $M)=\left(\bigcap_{P \in V(\mathcal{P} M)} P: M\right)$ immediately gives the desired equality $\mathcal{P}=(\sqrt[p]{\mathcal{P} M}: M)$.

Definition 2.2. Let $M$ be an $R$-module $M$. We call that $M$ is $\mathbb{P}$ radical whenever it satisfies one of the equivalent conditions listed in the above proposition.

The following proposition together with Example 2.6, below, asserts that the class of $\mathbb{P}$-radical is wider than that of primeful modules introduced in [19].

Proposition 2.3. Any primeful $R$-module $M$ is $\mathbb{P}$-radical.

Proof. Let $\mathcal{P} \supseteq \operatorname{Ann}(M)$ be a prime ideal. By the primeful assumption of $M$, there is some $P \in \operatorname{Spec}(M)$ with $(P: M)=\mathcal{P}$. It follows that $\sqrt[p]{\mathcal{P} M} \subseteq P$ and $\mathcal{P}=\sqrt{\mathcal{P}} \subseteq(\sqrt[p]{\mathcal{P} M}: M)$, and consequently we have:

$$
\mathcal{P}=\sqrt{\mathcal{P}} \subseteq(\sqrt[p]{\mathcal{P} M}: M) \subseteq(P: M)=\mathcal{P},
$$

which immediately implies that $(\sqrt[p]{\mathcal{P} M}: M)=\mathcal{P}$. Thus, $M$ is a $\mathbb{P}$-radical module. 
For an arbitrary ring $R$, it is easy to check that every free $R$-module $M$ is primeful. Moreover, it is shown that every finitely generated $R$ module $M$ and also every projective module over a domain is primeful (see [17, Theorem 2.2, Corollary 2.6]). Thus, we immediately obtain the following corollary.

Corollary 2.4. Let $R$ be a ring.

(i) Every free $R$-module is a $\mathbb{P}$-radical.

(ii) Every finitely generated $R$-module is a $\mathbb{P}$-radical.

(iii) If $R$ is a domain, then every projective $R$-module is a $\mathbb{P}$-radical.

We show that Corollary 2.4 (iii) is also true when we replace the phrase " $R$ is a domain" with " $R$ is a Noetherian ring."

Theorem 2.5. Every projective $R$-module is $\mathbb{P}$-radical whenever $R$ is a Noetherian ring.

Proof. Suppose that $M$ is a projective $R$-module and $\mathcal{P} \supseteq \operatorname{Ann}(M)$. We claim that $\mathcal{P} M \neq M$. Indeed, if $\mathcal{P} M=M$, then the collection $\mathcal{A}=\{I \unrhd R \mid I M \neq M$ and $\operatorname{Ann}(M) \subseteq I \subseteq \mathcal{P}\}$ is not empty since $\operatorname{Ann}(M) \in \mathcal{A}$. The Noetherian assumption of $R$ implies that $\mathcal{A}$ has a maximal element, say $\mathcal{P}_{0}$. If $\mathcal{P}_{0}$ is not a prime ideal of $R$, then there exist $a, b \in R \backslash \mathcal{P}_{0}$ such that $a b \in \mathcal{P}_{0}$. It follows that $\left(\mathcal{P}_{0}+R a\right) M=$ $\left(\mathcal{P}_{0}+R b\right) M=M$, and so $M=\left(\mathcal{P}_{0}+R a\right)\left(\mathcal{P}_{0}+R b\right) M \subseteq \mathcal{P}_{0} M$, which is a contradiction. Since for each projective $R$-module $M$ and each ideal $I$ of $R$, the factor module $M / I M$ is also projective as an $R / I$-module, thus $\bar{M}:=M / \mathcal{P}_{0} M$ is projective as an $\bar{R}:=R / \mathcal{P}_{0}$-module. Furthermore, $\bar{R}$ is a domain, and hence $\bar{M}$ is a $\mathbb{P}$-radical $\bar{R}$-module according to Corollary 2.4 (iii). Now, if $r \in R \backslash \mathcal{P}_{0}$, then $\left(R r+\mathcal{P}_{0}\right) M=M$, and it follows that $r+\mathcal{P}_{0} \notin \operatorname{Ann}_{\bar{R}}(\bar{M})$, i.e., $\operatorname{Ann}_{\bar{R}}(\bar{M})=(0)$. Since $\overline{\mathcal{P}}:=\mathcal{P} / \mathcal{P}_{0}$ is a prime ideal of $\bar{R}$, we must have $(\sqrt[p]{\overline{\mathcal{P} M}}: \bar{M})=\overline{\mathcal{P}}$. But, the equality $\mathcal{P} M=M$ implies that $\overline{\mathcal{P} M}=\bar{M}$, and so $(\sqrt[p]{\overline{\mathcal{P} M}}: \bar{M})=R$, which is a contradiction. Thus, $\mathcal{P} M \neq M$ and so $\mathcal{P} M$ is a proper submodule of $M$. Suppose $F=M \oplus L$ where $F$ is a free $R$-module and $L$ is a submodule of $F$. Clearly, $\mathcal{P} F$ is a prime submodule of $F$, namely, $F / \mathcal{P} F$ is a prime $R$-module and $\operatorname{Ann}(F / \mathcal{P} F)=\mathcal{P}$. Since $F / \mathcal{P} F \cong M / \mathcal{P} M \oplus L / \mathcal{P} L$, we conclude that $M / \mathcal{P} M$ is also 
a prime $R$-module with $\operatorname{Ann}(M / \mathcal{P} M)=\operatorname{Ann}(F / \mathcal{P} F)=\mathcal{P}$, and hence $\mathcal{P} M$ is a prime submodule of $M$ with $(\mathcal{P} M: M)=\mathcal{P}$. Thus, $(\sqrt[p]{\mathcal{P} M}: M)=(\mathcal{P} M: M)=\mathcal{P}$, which implies satisfaction of the $\mathbb{P}$-radical condition on the $R$-module $M$.

The following example shows that the converse of Proposition 2.3 is not true in general and, consequently, the class of $\mathbb{P}$-radical modules contains the class of primeful $R$-modules, properly. Moreover, the next proposition helps us to better recognize the relationship between these two types of modules.

Example 2.6. (see also [19, page 136, Example 1]). Consider the $\mathbb{Z}$-module $M:=\bigoplus_{p \in \Omega} \mathbb{Z} / p \mathbb{Z}$ for the set of prime integers $\Omega$. One easily convinces oneself that Ann $(M)=0$ and according to [19], for any nonzero prime ideal $(p)$ of $\mathbb{Z}, p M$ is a $(p)$-prime submodule of $M$, while it doesn't have any (0)-prime submodule. Consequently, $M$ is not a primeful $\mathbb{Z}$-module. The zero submodule $(0)$ of $M$ is an intersection of maximal submodules and hence $\sqrt[p]{(0)}=(0)$. Thus:

$$
(\sqrt[p]{(0) M}: M)=((0): M)=\operatorname{Ann}(M)=(0) .
$$

Furthermore, if $(q)$ is a non-zero prime (maximal) ideal of $\mathbb{Z}$, then $(q) M=\bigoplus_{q \neq p \in \Omega} \mathbb{Z} / p \mathbb{Z} \neq M$. It follows that $(q)=(\sqrt[p]{(q) M}: M)$, and hence $M$ is $\mathbb{P}$-radical.

Proposition 2.7. For every ring $R$, there is a non-primeful $\mathbb{P}$-radical $R$-module $M$ if and only if there exist some prime ideals $\mathcal{P}$ and $\left\{\mathcal{P}_{i}\right\}_{i \in I}$ of $R$ such that $\mathcal{P} \subset \mathcal{P}_{i}$ and $\mathcal{P}=\bigcap_{i \in I} \mathcal{P}_{i}$.

Proof. Let $M$ be a non-primeful $\mathbb{P}$-radical $R$-module and $\mathcal{P}$ a prime ideal of $R$ such that $M$ does not have any $\mathcal{P}$-prime submodules. Then, for any prime submodule $N$ of $M$ with $\mathcal{P} M \subseteq N$, we should have $\mathcal{P} \subset \mathcal{P}_{N}:=(N: M)$. On the other hand, the $\mathbb{P}$-radical property of $M$ implies that:

$$
\begin{aligned}
\mathcal{P} & =(\sqrt[p]{\mathcal{P} M}: M)=\left(\bigcap_{N \in X} N: M\right) \\
& =\bigcap_{N \in X}(N: M)=\bigcap_{N \in X} \mathcal{P}_{N},
\end{aligned}
$$


where $X=\{N \mid N \in \operatorname{Spec}(M)$ with $\mathcal{P} M \subseteq N\}$. For the converse, there is no loss of generality in assuming that $\mathcal{P}=\bigcap_{i \in I} \mathcal{P}_{i}$ and, for each prime ideal $\mathcal{Q} \supset \mathcal{P}$ of $R$, there exists $i \in I$ such that $\mathcal{Q}=\mathcal{P}_{i}$. Consider the $R$-module $M=\bigoplus_{i \in I} R / \mathcal{P}_{i}$. Then, $\operatorname{Ann}(M)=\bigcap_{i \in I} \mathcal{P}_{i}=\mathcal{P}$ and, for each $j \in I, N_{j}=\bigoplus_{j \neq i \in I} R / \mathcal{P}_{i}$ is a $\mathcal{P}_{j}$-prime submodule of $M$ with $\mathcal{P} M \subseteq \mathcal{P}_{j} M \subseteq N_{j}$. Thus, for each prime ideal $\mathcal{P}_{j} \supset \operatorname{Ann}(M)=\mathcal{P}$, we have $\left(\sqrt[p]{\mathcal{P}_{j} M}: M\right)=\mathcal{P}_{j}\left(\right.$ since $\left.\mathcal{P}_{j} \subseteq\left(\sqrt[p]{\mathcal{P}_{j} M}: M\right) \subseteq\left(N_{j}: M\right)=\mathcal{P}_{j}\right)$. On the other hand,

$$
(\sqrt[p]{\mathcal{P} M}: M) \subseteq\left(\bigcap_{i \in I} N_{i}: M\right)=\bigcap_{i \in I}\left(N_{i}: M\right)=\bigcap_{i \in I} \mathcal{P}_{i}=\mathcal{P},
$$

and hence $M$ is a $\mathbb{P}$-radical module. We claim that $M$ doesn't have any $\mathcal{P}$-prime submodule. Otherwise, let $N$ be a $\mathcal{P}$-prime submodule of $M$ with $(N: M)=\mathcal{P}$. Since $N \neq M$, there exists $j \in I$ such that $\left(\cdots, 0,1+\mathcal{P}_{j}, 0, \cdots\right) \notin N$. Since $\mathcal{P}_{j}\left(\cdots, 0,1+\mathcal{P}_{j}, 0, \cdots\right) \in N$, we should have $\mathcal{P}_{j} M \subseteq N$, namely, $\mathcal{P}_{j} \subseteq \mathcal{P}$, which is a contradiction. Thus, $M$ is not primeful.

According to Proposition 2.1, an $R$-module $M$ is $\mathbb{P}$-radical if and only if $(\sqrt[p]{\mathcal{P} M}: M)=\mathcal{P}$ for each prime ideal $\mathcal{P} \supseteq \operatorname{Ann}(M)$. Now let us generalize it by the notion of $\mathbb{M}$-radical modules.

Definition 2.8. An $R$-module $M$ is called $\mathbb{M}$-radical (or Maxful) whenever $(\sqrt[p]{\mathcal{M} M}: M)=\mathcal{M}$ for each maximal ideal $\mathcal{M} \supseteq \operatorname{Ann}(M)$.

The following evident proposition offers several other characterizations of $\mathbb{M}$-radical modules.

Proposition 2.9. The following statements are equivalent for a nonzero $R$-module $M$ :

(1) $M$ is an $\mathbb{M}$-radical module.

(2) $\mathcal{M} M \neq M$ for every maximal ideal $\mathcal{M} \supseteq \operatorname{Ann}(M)$.

(3) $\mathcal{P} M \neq M$ for every prime ideal $\mathcal{P} \supseteq \operatorname{Ann}(M)$.

(4) There is a maximal submodule $P$ of $M$ such that $(P: M)=\mathcal{M}$ for every maximal ideal $\mathcal{M} \supseteq \operatorname{Ann}(M)$.

(5) There is a prime submodule $P$ of $M$ such that $(P: M)=\mathcal{M}$ for every maximal ideal $\mathcal{M} \supseteq \operatorname{Ann}(M)$. 
Proof. (1) $\Rightarrow(2)$ can be immediately obtained from the direct definition of the M-radical modules.

For $(2) \Rightarrow(1)$, suppose that $\mathcal{M} M \neq M$ for every maximal ideal $\mathcal{M} \supseteq \operatorname{Ann}(M)$. Then, for each maximal ideal $\mathcal{M} \supseteq \operatorname{Ann}(M), \mathcal{M} M$ is a prime submodule of $M$, and so $\sqrt[p]{\mathcal{M} M}=\mathcal{M} M$. It follows that $\mathcal{M} \subseteq$ $(\sqrt[p]{\mathcal{M M}}: M)=(\mathcal{M} M: M)=\mathcal{M}$, and hence $(\sqrt[p]{\mathcal{M} M}: M)=\mathcal{M}$, as desired.

The case $(2) \Leftrightarrow(3)$ is obvious.

For $(2) \Rightarrow(5)$, suppose that $\mathcal{M} \supseteq \operatorname{Ann}(M)$ is a maximal ideal. Then the non-equality $\mathcal{M} M \neq M$ implies that $\mathcal{M} M$ is a prime submodule with $(\mathcal{M} M: M)=\mathcal{M}$.

Two cases, (5) $\Rightarrow(2)$ and (4) $\Rightarrow(5)$, are immediate and for (5) $\Rightarrow(4)$, suppose that $(P: M)=\mathcal{M}$ where $P$ is a prime submodule of $M$ and $\mathcal{M} \supseteq \operatorname{Ann}(M)$ is a maximal. Then $M / P$ is an $R / \mathcal{M}$-vector space, and hence $M / P$ has a maximal $R / \mathcal{M}$-subspace such as $K / P$. Then, $K \subseteq M$ is a maximal $R$-submodule and $(K: M)=\mathcal{M}$, as desired.

A commutative ring $R$ is called a Hilbert ring-or Jacobson or Jacobson-Hilbert ring-if every prime ideal of $R$ is an intersection of its maximal ideals. The class of commutative Hilbert rings is closed under the finite polynomial rings forming. On the other hand, we have already observed some modules $M$ having no prime submodule (for example $\mathbb{Z}_{p^{\infty}}$ as a $\mathbb{Z}$-module). We call such modules primeless. We recall that a module $M$ over a domain $R$ is called divisible if $r M=M$ for each $0 \neq r \in R$ and is called torsion if $\operatorname{Ann}(m) \neq 0$ for each $m \in M$. We shall be interested in seeing, under which conditions, two notions of $\mathbb{P}$-radical and $\mathbb{M}$-radical are equivalent. We characterize this equivalency using the notion of Hilbert rings. But, at first, we need the following lemma.

Lemma 2.10. (see [23, Lemma 1.3 (i)]). Let $R$ be a domain. Then every torsion divisible $R$-module is primeless.

Theorem 2.11. For an arbitrary ring $R$, every $\mathbb{M}$-radical $R$-module is $\mathbb{P}$-radical if and only if $R$ is a Hilbert ring. 
Proof. Let every $\mathbb{M}$-radical $R$-module be $\mathbb{P}$-radical. To obtain a contradiction, suppose that $\mathcal{P}$ is a prime ideal of $R$ which is not an intersection of the maximal ideals of $R$. One convinces oneself that every $\mathbb{M}$-radical $R / \mathcal{P}$-module is also a $\mathbb{P}$-radical $R / \mathcal{P}$-module. Let $S=R / \mathcal{P}$ and $Q$ be the field of fraction of $S$. Since $\mathcal{P}$ is not a maximal ideal, $S$ is not a field and so $S \neq Q$. For a non-zero proper $S$-submodule $K$ of $Q$ the quotient $L:=Q / K$ is a torsion divisible $S$-module and so, by the above lemma, $L$ is a primeless $S$-module. Now consider the $S$-module:

$$
M=\bigoplus_{\mathcal{M} \in \operatorname{Max}(S)} S / \mathcal{M} \oplus L .
$$

Since, for each $\mathcal{M}_{1} \in \operatorname{Max}(S)$, we have:

$$
\mathcal{M}_{1} M=\bigoplus_{\mathcal{M}_{1} \neq \mathcal{M} \in \operatorname{Max}(S)} S / \mathcal{M} \oplus L
$$

is a prime $S$-module with $\left(\mathcal{M}_{1} M: M\right)=\mathcal{M}_{1}$, then $M$ is $\mathbb{M}$-radical. We claim that every prime $S$-submodule of $M$ is also of the above form. To see this, let $P$ be a prime $S$-submodule of $M$. We have $\bigoplus_{\mathcal{M} \in \operatorname{Max}(S)} S / \mathcal{M} \nsubseteq P$; otherwise, we must have $M / P \cong L / T$ for some proper submodule $T$ of $L$, which is a contradiction according to the primeless assumption of $L$. Then there is some $\mathcal{M}_{1} \in \operatorname{Max}(S)$ such that $\left(0, \cdots, 0,1+\mathcal{M}_{1}, 0 \cdots\right) \notin P$. Since $\mathcal{M}_{1}\left(0, \cdots, 0,1+\mathcal{M}_{1}, 0 \cdots\right) \subseteq P$, hence $\mathcal{M}_{1} M \subseteq N$, and consequently, we have $\mathcal{M}_{1} M=N$. It follows that:

$$
\sqrt[p]{(0)}=\bigcap_{\mathcal{M} \in \operatorname{Max}(R)} \mathcal{M} M=L .
$$

Now the equality Ann $\left({ }_{S} L\right)=(0)$ implies that $\operatorname{Ann}\left({ }_{S} M\right)=(0)$ and hence:

$$
\begin{aligned}
(\sqrt[p]{(0) M}: M) & =(\sqrt[p]{(0)}: M)=(L: M)= \\
& =\left((0): \bigoplus_{\mathcal{M} \in \operatorname{Max}(S)} S / \mathcal{M}\right)=\bigcap_{\mathcal{M} \in \operatorname{Max}(R)} \mathcal{M} .
\end{aligned}
$$

But $\bigcap_{\mathcal{M} \in \operatorname{Max}(R)} \mathcal{M} \neq(0)$ since $\mathcal{P} \neq \bigcap_{\mathcal{P} \subseteq \mathcal{M} \in \operatorname{Max}(R)} \mathcal{M}$. Thus, $(\sqrt[p]{(0) M}: M) \neq(0)$, which is impossible (since $M$ is a $\mathbb{P}$-radical module). Thus, $R$ is a Hilbert ring. 
For the converse, assume that $M$ is an $\mathbb{M}$-radical module and $\mathcal{P}$ is a prime ideal of $R$ with $\mathcal{P} \supseteq \operatorname{Ann}(M)$. Since $R$ is a Hilbert ring, $\mathcal{P}=\bigcap_{\mathcal{P} \subseteq \mathcal{M} \in \operatorname{Max}(R)} \mathcal{M}$. The $\mathbb{M}$-radical property of $M$ implies that $(\mathcal{M} M: M)=\mathcal{M}$ for each $\mathcal{P} \subseteq \mathcal{M} \in \operatorname{Max}(R)$, and hence we have:

$$
\begin{aligned}
(\sqrt[p]{\mathcal{P} M}: M) & \subseteq\left(\bigcap_{\mathcal{P} \subseteq \mathcal{M} \in \operatorname{Max}(R)} \mathcal{M} M: M\right) \\
& =\bigcap_{\mathcal{P} \subseteq \mathcal{M} \in \operatorname{Max}(R)}(\mathcal{M} M: M) \\
& =\bigcap_{\mathcal{P} \subseteq \mathcal{M} \in \operatorname{Max}(R)} \mathcal{M}=\mathcal{P} .
\end{aligned}
$$

This inequality, together with the fact $\mathcal{P} \subseteq(\mathcal{P} M: M) \subseteq(\sqrt[p]{\mathcal{P} M}: M)$, implies that $(\sqrt[p]{\mathcal{P} M}: M)=\mathcal{P}$, as desired.

According to the results obtained so far, we have the following chart of implications for an arbitrary $R$-module $M$ :

$$
M \text { is primeful } \Rightarrow M \text { is } \mathbb{P} \text {-radical } \Rightarrow M \text { is } \mathbb{M} \text {-radical }
$$

Moreover, none of the implications is reversible in general. However, for zero-dimensional rings, we have the following result, which expresses the relationship between primeful and $\mathbb{M}$-radical modules.

Theorem 2.12. For an arbitrary ring $R$, every $\mathbb{M}$-radical module is also primeful if and only if $\operatorname{dim}(R)=0$.

Proof. If every $\mathbb{M}$-radical $R$-module is primeful, then, according to Theorem 2.11, $R$ is a Hilbert ring. Suppose, contrary to our claim, that $\operatorname{dim}(R) \geq 2$ and $\mathcal{P}$ is a non-maximal prime ideal of $R$. Thus, $\mathcal{P}=\bigcap_{\mathcal{P} \subseteq \mathcal{M} \in \operatorname{Max}(R)} \mathcal{M}$, and according to Proposition 2.7 there exists an $R$-module $M$ which is $\mathbb{P}$-radical but not primeful, a contradiction. Thus, $\operatorname{dim}(R)=0$. The converse is clear.

We shall be interested in seeing whether every $R$-module is primeful (and hence $\mathbb{P}$-radical and $\mathbb{M}$-radical). This question leads us to the concept of Artinian rings. 
Theorem 2.13. For an Artinian ring $R$, every $R$-module is primeful (and hence $\mathbb{P}$-radical and $\mathbb{M}$-radical).

Proof. We know that the dimension of the Artinian rings is identically zero and hence the three concepts of primeful, $\mathbb{P}$-radical and M-radical are equivalent for all modules defined over such rings. Since $R$ is Artinian, we can express it by:

$$
R=R_{1} \times \cdots \times R_{n}, \quad n \in \mathbb{N},
$$

where each $R_{i}$ is an Artinian local ring. In the special case $n=1$, where $R$ is a local ring with maximal ideal $\mathcal{M}$, consider the non-zero $R$-module $M$. Then Ann $(M) \neq R$, and so $\operatorname{Ann}(M) \subseteq \mathcal{M}$. By Proposition 2.9, it is sufficient to show that $\mathcal{M} \neq M$. If $R$ is a domain (field), then $\mathcal{M}=(0)$, and so the proof is complete. Hence, to obtain a contradiction, suppose that $R$ is not a domain and also $\mathcal{M} M=M$. Then there are some non-zero elements $a, b \in R$ such that $a b=0$. Thus, $(R a)(R b) M=(0)$, and consequently we should have either $R a M \neq M$ or $R b M \neq M$. It follows that $\mathcal{A}:=\{I \unrhd R \mid I M \neq M$ and $I \neq(0)\}$ is a non-empty set of the ideals of $R$. Since $R$ is Noetherian, $\mathcal{A}$ has a maximal element such as $\mathcal{P}$ which is not a prime (maximal) ideal of $R$ according to the inequality $\mathcal{P} M \neq M$. Thus, there exist two ideals $A$ and $B$ of $R$ such that $\mathcal{P} \subset A, \mathcal{P} \subset B$ and $A B \subseteq \mathcal{P}$. Now, using the equalities $A M=B M=M$ implies that $M=A B M \subseteq \mathcal{P} M$, a contradiction. Now, assume that $n \geq 2$, and for each $i(1 \leq i \leq n)$ let $\mathcal{M}_{i}$ be the maximal ideal of the local ring $R_{i}$. Furthermore, consider the non-zero $R$-module $M$ with $\operatorname{Ann}(M) \subseteq \mathcal{M}$ where $\mathcal{M}$ is a maximal ideal of $R$. Then $\mathcal{M}$ is of the form $R_{1} \times \cdots \times R_{i-1} \times \mathcal{M}_{i} \times R_{i+1} \cdots \times R_{n}$ for some $1 \leq i \leq n$. Without loss of generality, we can assume that $i=1$. Again, according to Proposition 2.9, it is sufficient to show that the inequality $\mathcal{M} M=\left(\mathcal{M}_{1} \times R_{2} \times \cdots \times R_{n}\right) M \neq M$ holds. On the contrary, suppose that $\left(\mathcal{M}_{1} \times R_{2} \times \cdots \times R_{n}\right) M=M$. Consider two ideals $I=R_{1} \times(0) \times \cdots \times(0)$ and $J=(0) \times R_{2} \times \cdots \times R_{n}$ of $R$. We have $J=\operatorname{Ann}(I)$, and hence $R_{1} \cong R / J$ which implies that $\bar{M}=I M$ is a unitary $R_{1}$-module (in fact, $\bar{M}=\left(R_{1} \times 0 \times \cdots \times 0\right) M$ is a unitary $R_{1}$-module with $r_{1} \bar{m}$ defined to be $r_{1}(1,0, \cdots, 0) \bar{m}$ for $r_{1} \in R_{1}$ and $\bar{m} \in \bar{M})$. We claim that $\bar{M} \neq(0)$, otherwise, $R_{1} \times(0) \times \cdots \times(0) \subseteq$ $\operatorname{Ann}(M) \subseteq \mathcal{M}_{1} \times R_{2} \times \cdots \times R_{n}$, which is a contradiction. Thus, $\bar{M}$ is a non-zero $R_{1}$-module and so, according to the case $n=1$, we have $\mathcal{M}_{1} \bar{M} \neq \bar{M}$, i.e., $\left(\mathcal{M}_{1} \times 0 \times \cdots \times 0\right) M \neq\left(R_{1} \times 0 \times \cdots \times 0\right) M$. 
On the other hand, for each $m \in M$, we have $(1,0, \cdots, 0) m \in M=$ $\left(\mathcal{M}_{1} \times R_{2} \times \cdots \times R_{n}\right) M$ and, consequently, for each $m \in M$, we have:

$$
(1,0, \ldots, 0) m=\sum_{j=1}^{k}\left(p_{1 j}, r_{2 j}, \ldots, r_{n j}\right) m_{j},
$$

where $k \in \mathbb{N}, m_{j} \in M, p_{1 j} \in \mathcal{M}_{1}$ and $r_{i j} \in R_{i}$. Thus:

$$
\begin{aligned}
(1,0, \ldots, 0) m & =(1,0, \ldots, 0)^{2} m \\
& =\sum_{j=1}^{k}\left(p_{1 j}, 0, \ldots, 0\right) m_{j},
\end{aligned}
$$

and hence $(1,0, \ldots, 0) m \in\left(\mathcal{M}_{1} \times(0) \times \cdots \times(0)\right) M$, for each $m \in M$. It follows that $\left(R_{1} \times 0 \times \cdots \times 0\right) M \subseteq\left(\mathcal{M}_{1} \times(0) \times \cdots \times(0)\right) M$, i.e., $\mathcal{M}_{1} \bar{M}=\bar{M}$, which is a contradiction.

The following example shows that the converse of the above theorem is not true, in general.

Example 2.14. Let $K$ be a field, $D:=K\left[\left\{x_{i}: i \in \mathbb{N}\right\}\right]$ (a unique factorization domain) and $R=K\left[\left\{x_{i}: i \in \mathbb{N}\right\}\right] /\left(\left\{x_{i} x_{j}: i, j \in \mathbb{N}\right\}\right)$, where $\left(\left\{x_{i} x_{j}: i, j \in \mathbb{N}\right\}\right)$ is the ideal of $D$ generated by $\left\{x_{i} x_{j}: i, j \in\right.$ $\mathbb{N}\} \subseteq D$. Furthermore, for each $k \in \mathbb{N}$, let $\bar{x}_{k}=x_{k}+\left(\left\{x_{i} x_{j}: i, j \in \mathbb{N}\right\}\right)$ and $\mathcal{M}=\left(\left\{\bar{x}_{k}: k \in \mathbb{N}\right\}\right)$. The ideal $\mathcal{M}$ is simply the image of the maximal ideal $\mathcal{N}=\left(\left\{x_{k}: k \in \mathbb{N}\right\}\right)$ of $D$. Clearly $\mathcal{M}^{2}=(0)$, and so $R$ is a local zero-dimensional ring, but is not Artinian (Noetherian). The equality $\mathcal{M}^{2}=(0)$ implies that, for each non-zero $R$-module, we have $M, \mathcal{M} M \neq M$, and hence according to Proposition 2.9 and Theorem 2.12, $M$ is a $\mathbb{P}$-radical module. Thus, every $R$-module is $\mathbb{P}$ radical, while $R$ is not Artinian.

A ring $R$ is called a Max-ring (or a Bass ring) if every non-zero $R$-module has a maximal submodule. Also, a ring $R$ is called a $P$-ring if every non-zero $R$-module has a prime submodule. It is proved that the commutative P-rings coincide with the Max-rings (see [7, Theorem 3.9]). Moreover, we have the following lemma.

Lemma 2.15. (see [14, Theorem 2]). For a commutative ring $R$, the following conditions are equivalent: 
(1) $R$ is a max ring;

(2) $R / J(R)$ is a regular ring and $J(R)$ is a t-nilpotent ideal.

The following theorem offers several characterizations of Noetherian rings $R$ over which every module is $\mathbb{P}$-radical.

Theorem 2.16. Consider the following statements for a ring $R$ :

(1) $R$ is an Artinian ring.

(2) Every $R$-module is primeful.

(3) Every $R$-module is $\mathbb{P}$-radical.

(4) Every $R$-module is $\mathbb{M}$-radical.

(5) $R$ is a Max-ring.

(6) $R$ is a P-ring.

(7) $\operatorname{dim}(R)=0$.

Then $(1) \Rightarrow(2) \Leftrightarrow(3) \Leftrightarrow(4) \Rightarrow(5) \Leftrightarrow(6) \Rightarrow(7)$. Moreover, when $R$ is Noetherian (or domain), all the seven statements are equivalent.

Proof. (1) $\Rightarrow(2)$ is Theorem 2.13 .

$(2) \Rightarrow(3)$ is Proposition 2.3. (3) $\Rightarrow(4)$ is clear.

$(4) \Rightarrow(5)$. Assume that every $R$-module is $\mathbb{M}$-radical. For an arbitrary non-zero $R$-module $M$ we have $\operatorname{Ann}(M) \neq R$ and so there is a maximal ideal $\mathcal{M}$ of $R$ such that $\operatorname{Ann}(M) \subseteq \mathcal{M}$. Since $M$ is maxful, there is, moreover, a prime submodule $P$ of $M$ with $(P: M)=\mathcal{M}$. Thus, $M / P$ is an $R / \mathcal{M}$-module $(R / \mathcal{M}$-vector space), and hence, $M / P$ has a maximal $R / \mathcal{M}$-submodule such as $K / P$. It is easy to see that $K<M$ is a maximal $R$-submodule. Thus, every non-zero $R$-module has a maximal submodule, namely, $R$ is a Max-ring.

$(5) \Leftrightarrow(6)$ is by [7, Theorem 3.9].

$(6) \Rightarrow(7)$. Let $R$ be a P-ring and $\mathcal{P}$ a non-maximal prime ideal of $R$. For $R^{\prime}:=R / \mathcal{P}$, consider $K$ as the field of fractions of $R^{\prime}$. Since $R^{\prime}$ is not a field, then $R^{\prime} \neq K$, and $K$ is a divisible $R^{\prime}$-module. It follows that $K / R^{\prime}$ is a non-zero torsion divisible $R^{\prime}$-module. Then, according to Lemma $2.10, K / R^{\prime}$ is a primeless $R^{\prime}$-module. Now, one convinces oneself that $K / R^{\prime}$ is a primeless $R$-module and consequently $R$ is not a P-ring, which is a contradiction.

$(4) \Rightarrow(2)$. If (4) holds, one concludes from $(4) \Rightarrow(7)$ that $\operatorname{dim}(R)=0$. Then, according to Theorem 2.12 , every $R$-module is primeful. 
Finally, if $R$ is a Noetherian ring, then $\operatorname{dim}(R)=0$ if and only if $R$ is Artinian. Thus, $(7) \Rightarrow(1)$ holds whenever $R$ is a Noetherian ring.

The following is now immediate.

Corollary 2.17. For a domain $R$, the following statements are equivalent:

(1) Every $R$-module is primeful.

(2) Every $R$-module is $\mathbb{P}$-radical.

(3) Every $R$-module is $\mathbb{M}$-radical.

(4) $R$ is a field (i.e., $R$ is an Artinan domain).

The following proposition demonstrates the relationship between finitely generated, primeful, $\mathbb{P}$-radical and $\mathbb{M}$-radical modules in the interesting case of multiplication modules.

Proposition 2.18. Consider the following statements for a non-zero $R$-module $M$ :

(1) $M$ is finitely generated.

(2) $M$ is primeful.

(3) $M$ is a $\mathbb{P}$-radical module.

(4) $(\mathcal{P} M: M)=\mathcal{P}$ for every prime ideal $\mathcal{P} \supseteq \operatorname{Ann}(M)$.

(5) $M$ is a $\mathbb{M}$-radical module.

Then $(1) \Rightarrow(2) \Rightarrow(3) \Rightarrow(4) \Rightarrow(5)$. In particular, when $M$ is a multiplication module, then (5) $\Rightarrow(1)$ holds, too.

Proof. (1) $\Rightarrow(2)$ holds by [19, Proposition 3.8], and (2) $\Rightarrow(3)$ holds by Proposition 2.3. Moreover, $(3) \Rightarrow(4)$ is straightforward by the fact $\mathcal{P} \subseteq(\mathcal{P} M: M) \subseteq(\sqrt[p]{\mathcal{P} M}: M)$ for every prime ideal $\mathcal{P} \supseteq \operatorname{Ann}(M)$. To prove $(4) \Rightarrow(5)$, we know that the equality $(\mathcal{P} M: M)=\mathcal{P}$ implies that $\mathcal{P} M \neq M$ for every prime ideal $\mathcal{P} \supseteq \operatorname{Ann}(M)$. Thus, $M$ is an M-radical module. When $M$ is a multiplication module, then (5) $\Rightarrow(1)$ holds according to [19, Proposition 3.8]. This completes the proof.

We conclude this section with the next analogue of Nakayama's lemma. 
Proposition 2.19. Let $M$ be an $\mathbb{M}$-radical $R$-module. Then $M$ satisfies the following assertion (NAK): If $I$ is an ideal of $R$ contained in the Jacobson radical $J(R)$ with $I M=M$, then $M=(0)$.

Proof. If $M \neq(0)$ then, $\operatorname{Ann}(M) \neq R$. Hence, for a maximal ideal $\mathcal{M}$ of $R$ containing Ann $(M)$, we have $I \subseteq \mathcal{M}$ and $I M=M=\mathcal{M} M$, which is a contradiction.

3. Characterization of semisimple $\mathbb{P}$-radical modules. Recall that, for an $R$-module module $M$, the socle of $M$ (denoted by $\operatorname{soc}(M)$ is the sum of all simple (minimal) submodules of $M$. If there are no minimal submodules in $M$, we put $\operatorname{soc}(M)=(0)$. Thus, $M$ is a semisimple module whenever $\operatorname{soc}(M)=M$. Furthermore, a semisimple module $M$ is called homogeneous if any two simple submodules of $M$ are isomorphic. One checks that an $R$-module $M$ is homogeneous semisimple if and only if $\operatorname{Ann}(M)$ is a maximal ideal. In this section, we aim to characterize semisimple $\mathbb{P}$-radical modules. First we need the following definition.

Definition 3.1. Let $R$ be a ring. A semisimple $R$-module $M$ is called full semisimple if, for each maximal ideal $\mathcal{M} \supseteq \operatorname{Ann}(M)$ the simple $R$ module $R / \mathcal{M}$ can be embedded in $M$, namely, there exists a submodule $N$ of $M$ such that $N \cong \bigoplus_{\operatorname{Ann}(M) \subseteq \mathcal{M} \in \operatorname{Max}(R)} R / \mathcal{M}$.

Example 3.2. Consider the $\mathbb{Z}$-module $M=\bigoplus_{p \in \Omega} \mathbb{Z} / p \mathbb{Z}$ where $\Omega$ is the set of prime integers. Obviously, $M$ is a full semisimple $\mathbb{Z}$ module while the semisimple $\mathbb{Z}$-module $M_{1}=\bigoplus_{2 \neq p \in \Omega} \mathbb{Z} / p \mathbb{Z}$ is not full semisimple since $\operatorname{Ann}\left(M_{1}\right)=(0) \subseteq 2 \mathbb{Z}$ and $\mathbb{Z} / 2 \mathbb{Z}$ is not a submodule of $M_{1}$.

The proof of the following result is straightforward and left to the reader.

Proposition 3.3. Let $M$ be a semisimple R-module such that Ann $(M)$ is a finite intersection of maximal ideals. Then $M$ is full semisimple. In particular, all finitely generated semisimple modules are full semisimple as well as all homogenous semisimple modules. 
Lemma 3.4. Let $M$ be an $R$-module with the non-zero socle. Then $M$ is a prime module if and only if $M$ is a homogeneous semisimple module.

Proof. Let $M$ be a prime module with non-zero socle, and let $R m$ be a simple submodule of $M$ for some $m \in M$. Then $\operatorname{Ann}(m)=$ $\operatorname{Ann}(M)=P$, and hence $P$ is a maximal ideal of $R$. Since Ann $(m)=$ Ann $\left(m^{\prime}\right)$ for each $0 \neq m^{\prime} \in M$, then $M$ is a homogeneous semisimple $R$-module. The converse is evident.

We are now in a position to show that the two concepts of M-radical and full semisimple are equivalent in the case of semisimple modules.

Proposition 3.5. Let $M$ be a semisimple $R$-module. Then $M$ is $\mathbb{M}$ radical if and only if $M$ is full semisimple.

Proof. Since $M$ is a semisimple $R$-module, we can assume that $M=\bigoplus_{i \in I} R / \mathcal{M}_{i}$ where $I$ is an index set and each $\mathcal{M}_{i}$ is a maximal ideal of $R$. Let $M$ be an $\mathbb{M}$-radical module. Then $\operatorname{Ann}(M)=\bigcap_{i \in I} \mathcal{M}_{i}$. Suppose that $\mathcal{M} \supseteq \operatorname{Ann}(M)$ is a maximal ideal of $R$. If $\mathcal{M} \neq \mathcal{M}_{i}$, then, $\mathcal{M}\left(R / \mathcal{M}_{i}\right)=R / \mathcal{M}_{i}$ for each $i \in I$. It follows that $\mathcal{M} M=M$, which is in contrary to Proposition 2.9 (2). Thus, $\mathcal{M}=\mathcal{M}_{i}$ for some $i \in I$ and hence $R / \mathcal{M}$ can be embedded in $M$. To prove the converse, assume that $\mathcal{M} \supseteq \operatorname{Ann}(M)$ is a maximal ideal of $R$. Since $M$ is full semisimple, then $\mathcal{M}=\mathcal{M}_{i}$ for each $i \in I$ and so $\mathcal{M} M \neq M$. Thus $M$ is an M-radical module, according to Proposition 2.9.

Proposition 3.6. For a semisimple $R$-module $M$, the following statements are equivalent:

(1) $M$ is a $\mathbb{P}$-radical module.

(2) $M$ is an $\mathbb{M}$-radical module and $R / \operatorname{Ann}(M)$ is a Hilbert ring.

(3) $M$ is full semisimple and $R / \operatorname{Ann}(M)$ is a Hilbert ring.

Proof. Since $M$ is a semisimple $R$-module, we can assume that $M=$ $\bigoplus_{i \in I} R / \mathcal{M}_{i}$ where $I$ is an index set and each $\mathcal{M}_{i}$ is a maximal ideal of $R$. To prove $(1) \Rightarrow(2)$, one should notice that since every $\mathbb{P}$-radical module is $\mathbb{M}$-radical, then it is sufficient to show that $R / \operatorname{Ann}(M)$ is a Hilbert ring. Suppose $\mathcal{P} \supseteq \operatorname{Ann}(M)$ is a prime ideal of $R$. Thus, we 
have $(\sqrt[p]{\mathcal{P} M}: M)=\mathcal{P}$ and hence $\sqrt[p]{\mathcal{P} M} \neq M$. We can assume that $\sqrt[p]{\mathcal{P} M}=\bigcap_{\lambda \in \Lambda} P_{\lambda}$, where $\Lambda$ is an index set and each $P_{\lambda}$ is a prime submodule of $M$ containing $\mathcal{P} M$. Hence, the factor module $M / P_{\lambda}$ is a prime semisimple module for each $P_{\lambda}$ and, consequently, $M / P_{\lambda}$ is a homogenous semisimple $R$-module, according to by Lemma 3.4. More precisely, $\mathcal{M}_{\lambda}:=\left(P_{\lambda}: M\right)$ is a maximal ideal of $R$. Thus:

$$
\mathcal{P}=(\sqrt[p]{\mathcal{P} M}: M)=\left(\bigcap_{\lambda \in \Lambda} P_{\lambda}: M\right)=\bigcap_{\lambda \in \Lambda}\left(P_{\lambda}: M\right)=\bigcap_{\lambda \in \Lambda} \mathcal{M}_{\lambda},
$$

and hence every prime ideal $\mathcal{P} \supseteq \operatorname{Ann}(M)$ is an intersection of maximal ideals of $R$, namely, $R / \operatorname{Ann}(M)$ is a Hilbert ring. $(2) \Leftrightarrow(3)$ and $(2) \Rightarrow(1)$ hold by Proposition 3.5 and Theorem 2.11, respectively.

Corollary 3.7. For a semisimple $R$-module $M$ where $R$ is either a Hilbert ring or a domain of dimension one, the following statements are equivalent:

(1) $M$ is $\mathbb{P}$-radical.

(2) $M$ is M-radical.

(3) $M$ is full semisimple.

Proof. If $R$ is a Hilbert ring then by Proposition 3.5 and Theorem 2.11, the proof is complete. Thus, we can assume that $R$ is a domain of dimension one. Since $M$ is a semisimple $R$-module, we can assume that $M=\bigoplus_{i \in I} R / \mathcal{M}_{i}$ where $I$ is an index set and each $\mathcal{M}_{i}$ is a maximal ideal of $R$. Now (1) $\Rightarrow(2)$ is straightforward and (2) $\Rightarrow(3)$ is Proposition 3.5. To prove $(3) \Rightarrow(1)$, assume that $\mathcal{P} \supseteq \operatorname{Ann}(M)$ is a prime ideal of $R$, and let $\mathcal{M} \supseteq \mathcal{P}$ be a maximal ideal. Since $R$ is a domain with $\operatorname{dim}(R)=1$, either $\mathcal{P}=(0)$ or $\mathcal{M}=\mathcal{P}$. If $\mathcal{M}=\mathcal{P}$, then $\mathcal{P}$ is one of the maximal ideals $\mathcal{M}_{i}$ in the direct summand of $M$ and so $\mathcal{P} M=\mathcal{M}_{i} M \neq M$. Clearly, $\mathcal{P} M$ is a prime submodule of $M$ with $(\mathcal{P} M: M)=\mathcal{P}$. It follows that $(\sqrt[p]{\mathcal{P} M}: M)=(\mathcal{P} M: M)=\mathcal{P}$. Otherwise, if $\mathcal{P}=(0)$ then, $\operatorname{Ann}(M)=(0)=\bigcap_{i \in I} \mathcal{M}_{i}$. We know that every proper submodule of a semisimple module is an intersection of maximal submodules and furthermore each maximal submodule is a prime submodule. Hence, $\sqrt[p]{\mathcal{P} M}=\sqrt[p]{(0)}=(0)$, which implies that $(\sqrt[p]{(0) M}: M)=((0): M)=\operatorname{Ann}(M)=(0)=\sqrt{(0)}$. Thus, $M$ is a $\mathbb{P}$-radical module. 
We conclude this paper with the following result that offers several characterizations for semisimple primeful modules.

Corollary 3.8. The following statements are equivalent for an arbitrary semisimple $R$-module $M$ :

(1) $M$ is primeful.

(2) $M$ is $\mathbb{P}$-radical and $\operatorname{dim}(R / \operatorname{Ann}(M))=0$.

(3) $M$ is $\mathbb{M}$-radical and $\operatorname{dim}(R / \operatorname{Ann}(M))=0$.

(4) $M$ is full semisimple and $\operatorname{dim}(R / \operatorname{Ann}(M))=0$.

Proof. To prove $(1) \Rightarrow(2)$, it is sufficient to show that $\operatorname{dim}(R /$ $\operatorname{Ann}(M))=0$. For a prime ideal $\mathcal{P} \supseteq \operatorname{Ann}(M)$ of $R$, there is a prime submodule $P$ of $M$ such that $(P: M)=\mathcal{P}$. Since $M / P$ is a prime semisimple $R$-module, then $\mathcal{P}$ is a maximal ideal according to Lemma 3.4. Thus, $\operatorname{dim}(R / \operatorname{Ann}(M))=0$. The assertion $(2) \Rightarrow$ (3) is a consequence of Theorem 2.12. Furthermore, (3) $\Rightarrow(4)$ is Proposition 3.5.

\section{REFERENCES}

1. M. Aghasi, M. Behboodi and M. Sabzevari, A structure sheaf on the spectrum of prime radical modules, J. Comm. Alg., to appear.

2. M.F. Atiyah and I. Macdonald, Introduction to commutative algebra, Addison-Wesley Pub. Co., 1969.

3. A. Barnard, Multiplication modules, J. Alg. 71 (1981), 174-178.

4. M. Behboodi, A generalization of the classical Krull dimension for modules, J. Alg. 305 (2006), 1128-1148.

5. _ A generalization of Bear's lower nilradical for modules, J. Alg. Appl. 6 (2007), 337-353.

6. On the prime radical and Baer's lower nilradical of modules, Acta Math. Hung. 122 (2009), 293-306.

7. M. Behboodi, O.A.S. Karamzadeh and H. Koohy, Modules whose certain submodules are prime, Vietnam J. Math. 32 (2004), 303-317.

8. B. Cortzen and L.W. Small, Finite extensions of rings, Proc. Amer. Math. Soc. 103 (1988), 1058-1062.

9. J. Dauns, Prime modules, J. reine angew. Math. 298 (1978), 156-181.

10. D. Eisenbud, Commutative algebra with a view toward algebraic geometry, Springer-Verlag, New York, 1995.

11. Z.A. El-Bast and P.F. Smith, Multiplication modules, Comm. Alg. 16 (1988), 755-779. 
12. E.H. Feller and E.W. Swokowski, Prime modules, Canad. J. Math. 17 (1965), 1041-1052.

13. O. Goldman, Hilbert rings and the Hilbert Nullstellensatz, Math. Z. 54 (1951), 136-140.

14. R. Hamsher, Commutative rings over which every module has a maximal submodule, Proc. Amer. Math. Soc. 18 (1967), 1133-1137.

15. R. Hartshorne, Algebraic geometry, Springer, New York, 1977.

16. C.P. Lu, Prime submodules of modules, Comm. Math. Univ. St. Paul 33 (1984), 61-69.

17. _ Spectra of modules, Comm. Alg. 23 (1995), 3741-3752.

18. The Zariski topology on the prime spectrum of a module, Houston J. Math. 25 (1999), 417-433.

19. A module whose prime spectrum has the suejective natural map, Houston J. Math. 33 (2007), 125-143.

20. _ M-radical of submodules in modules, Math. Japon. 34 (1989), 211219.

21. S.H. Man, One dimensional domains which satisfy the radical formula are Dedekind domains, Arch. Math. 66 (1996), 276-279.

22. R.L. McCasland and M.E. Moore, Prime submodules, Comm. Alg. 20 (1992), 1803-1817.

23. R.L. McCasland, M.E. Moore and P.F. Smith, On the spectrum of a module over a commutative ring, Comm. Alg. 25 (1997), 79-103.

24. R.L. McCasland and P.F. Smith, Prime submodules of Noetherian modules, Rocky Mountain J. Math. 23 (1993), 1041-1062.

25. P.F. Smith and D.P. Yilmaz, Radicals of submodules of free modules, Comm. Alg. 27 (1999), 2253-2266.

26. U. Tekir, On the sheaf of modules, Comm. Alg. 33 (2005), 2557-2562.

Department of Mathematical Sciences, Isfahan University of Technology, P.O.Box: 84156-83111, Isfahan, Iran, and School of Mathematics, Institute for Research in Fundamental Sciences (IPM), P.O.Box: 19395-5746, Tehran, IRAN

\section{Email address: mbehbood@cc.iut.ac.ir}

Department of Mathematics, University of Shahrekord, P. O. Box: 8818634141, Shahrekord, Iran, and School of Mathematics, Institute for ReSearch in Fundamental Sciences (IPM), P.O.Box: 19395-5746, Tehran, Iran Email address: sabzevari@math.iut.ac.ir 